\title{
El surgimiento del campo de la política educativa en Argentina: los casos de las Universidades Nacionales de Buenos Aires y La Plata
}

\author{
The educational policy field origins in Argentina: \\ the case of Buenos Aires and La Plata Federal \\ Universities
}

\section{O surgimento do campo da política educacional na Argentina: os casos das Universidades Nacionais de Buenos Aires e La Plata}

\author{
Julieta Susana Garcias Franco*
}

\begin{abstract}
Resumen: En este artículo se presentan resultados parciales de una investigación que se está desarrollando y cuyo objetivo consiste en mapear los enfoques y tendencias teóricas que asumió el campo teórico de la política educativa. Específicamente en este artículo se caracterizan y describen las tendencias teóricas del campo a partir de los programas de las cátedras de política educativa en la década de 1950 en las Universidades Nacionales de Buenos Aires y La Plata. Metodológicamente se analizaron 46 programas correspondientes al período 1917-1959 y desde allí, como paso sucesivo en la metodología, se indagaron las bibliografías de los programas del período de estudio y sus fundamentos teóricos vinculados a los profesores responsables de las cátedras de política educativa. De este modo y recurriendo a datos biográficos de los profesores se observa que en el período previo a la institucionalización del campo el debate se centraba en las tendencia teóricas del positivismo o antipositivismo. Dando lugar posteriormente a tendencias teóricas que se desprendían con distintos matices del positivismo y el antipositivismo de principios de siglo y que se entrecruzan en los programas desde 1953 a 1954, particularmente en tres ejes: tradición nacional, el progresismo pedagógico y krausismo español.
\end{abstract}

Palabras claves: Política Educativa. Surgimiento del campo. Tendencias teóricas.

Abstract: This paper presents partial results of an ongoing study whose objective is to map the approaches and theoretical trends in the educational policy theoretical field. More specifically, this study characterizes and describes the theoretical trends of the subject Educational Policy contents in the 50s of the last century in the Buenos Aires

*Universidad Nacional de Tres de Febrero (UNTREF). E-mail: <jgarciasfranco@gmail.com> 
and La Plata Federal Universities. 46 programs were analyzed in the period 1917-1959, as well as the bibliography and theoretical background linked to the professors who were responsible for the subject. Surveying professors' biographies, it was observed that in the period prior to the institutionalization of this field, the debate focused on the positivism or anti-positivism theoretical trends. Later on, the theoretical trends that permeated the programs from 1953 to 1954 had more distinct shades of positivism and anti-positivism than those of the beginning of the century, and three axes were observed: the national tradition, the pedagogical progressivism and the Spanish Krausism.

Keywords: Educational Policy. Field origin. Theoretical trends.

Resumo: Nesse artigo apresentam-se os resultados parciais de uma pesquisa em andamento cujo objetivo é mapear as abordagens e tendências teóricas do campo teórico da política educacional. Especificamente, nesse artigo, são caracterizadas e descritas as tendências teóricas dos programas das disciplinas de Política Educacional na década de 1950 nas Universidades Nacionais de Buenos Aires e La Plata. Foram analisados 46 programas do período 1917-1959, a partir dos quais foram analisadas as bibliografias e os seus fundamentos teóricos ligados aos professores responsáveis pela disciplina. Recorrendo a dados biográficos dos professores, observou-se que no período anterior à institucionalização do campo, o debate se centrava nas tendências teóricas do positivismo ou anti-positivismo. Posteriormente, isso levou a tendências teóricas que emergiram com matizes distintas de positivismo e anti-positivismo do início do século e que se entrecruzam nos programas desde 1953 a 1954, particularmente em três eixos: a tradição nacional, o progressismo pedagógico e o krausismo espanhol.

Palavras-chave: Política educacional. Surgimento do campo. Tendências teóricas.

\section{Presentación}

En este artículo presentamos los resultados parciales de una investigación ${ }^{1}$ que estamos llevando a cabo acerca de las tendencias teóricas del campo de la política educativa en Argentina, centrándonos en este caso en el período denominado surgimiento del campo (TELLO, 2013).

En esta investigación, observamos en primer término que el campo de la política educativa surge en Argentina en la década de 1950; esto se cristaliza con la creación de las cátedras del campo en las universidades nacionales de La Plata y Buenos Aires.

\footnotetext{
${ }^{1}$ Título: Surgimiento, construcción y estado actual de la política educativa como campo de estudio en Argentina. Código: UG087. CEIECS-UNSAM. Director: César Tello.
} 
En este sentido, debemos considerar que la creación de cátedras universitarias se convierte en una estructura que institucionaliza un campo emergente como lo fue el de la política educativa en la década de 1950. En términos de Bourdieu (2002), la formación de un nuevo campo intelectual surge como el producto de la propia historia que generó la autonomización de un sistema de conceptos para la enseñanza, la investigación y el desarrollo profesional.

También debemos tener en cuenta que, como lo afirman Gómez Campo y Tenti Fanfani (1989), los procesos de institucionalización de un determinado cuerpo de saberes, como es el caso del surgimiento de cátedras, generan un campo de estudio específico con mayor o menos estructuración, y así se inicia, de algún modo, un campo académico a partir del cual se desarrollan espacios de producción, circulación y validación de conocimientos, en el marco de una "trama histórica y dialéctica, a veces llena de contradicciones" (SIRVENT; RIGAL, 2007, p. 22).

En la indagación realizada observamos por primera vez el término "política educativa" en los espacios curriculares de las carreras en el año 1953 en la Universidad Nacional de La Plata cuya cátedra se denominó "Política Educacional y organización escolar" y con la misma denominación en la Universidad Nacional de Buenos Aires en el año 1955.

En este trabajo presentamos el análisis que hemos desarrollado de los programas curriculares del período 1953-1959. Para esto fue necesario hacer un rastreo bibliográfico, y considerando el período de análisis, donde los profesores se convierten en referentes académicos nacionales de las reflexiones y análisis sobre política educativa también hemos analizado los legajos de aquel período y sus propios escritos con la finalidad de comprender los principales enfoques teóricos que eran los ejes del escenario académico del período.

En la indagación que llevamos a cabo fue necesario realizar un rastreo de los programas desde los inicios de las carreras para localizar la incorporación del término "política educativa" como parte del título de algún espacio curricular. Por esta razón, inicialmente presentamos ese breve esbozo empírico a modo de contextualización de la historia biográfica de la investigación, de este modo, presentamos como secciones en este trabajo: a) Los programas precursores del campo; b) Surgimiento del campo: espacios curriculares de politica educativa, en primer término en la Universidad Nacional de La Plata y en segundo lugar en la Universidad Nacional de Buenos Aires para detenernos en la figura del profesor Gbioldi quien al ser docente que es nombrado de manera paralela en las dos casas de estudio en 1955 y permaneciendo en las cátedras de política educativa durante casi una década se estableció, de algún modo, como quien desarrolló las bases de la incorporación 
de la política educativa como espacio curricular en las carreras de ciencias de la educación.

\section{Los programas precursores del campo}

Sin pretender realizar una descripción exhaustiva de los programas precursores del campo, asunto que escapa al objetivo de este artículo, consideramos importante, señalar algunos rasgos histórico-ideológicos del campo donde se observan tensiones en cuanto a posiciones que los sostenían.

Debemos considerar que tanto en la UNLP como en la UBA desde principios de siglo ofrecían formación de docentes para las escuelas secundarias y las normales, pero la constitución de una carrera específica (Pedagogía o ciencias de la educación) recién se produjo a comienzos de la década de 1940 (PALAMIDESSI; GALARZA; CARDINI, 2012).

Desde fines del siglo XIX y en los principios del siglo XX el positivismo y el antipositivismo se disputaban las arenas de los diversos espacios académicos. Así nos encontramos en la Universidad Nacional de Buenos Aires con figuras de fuerte raigambre positivista como Carlos Octavio Bunge a cargo de "Ciencia y educación" " en 1917 y 1918 o Juan Ramos responsable de esa cátedra en 1922 o Antonio Fasce, que entre los años 1946 a 1954, fue el titular del seminario llamado "Organización Escolar". Por otro lado, y ubicándose en contraposición al positivismo filosófico de la época se encuentran Horacio Rivarola que en los años 1919 y 1920 fue profesor del espacio curricular “Ciencias de la Educación”, también en la Universidad Nacional de Buenos Aires y a Juan Cassani que además de ocupar distintos cargos en ambas universidades fue profesor del seminario "Legislación Escolar" a partir de 1938 en la UNLP y "Organización Escolar" en la UBA entre los años 1946-1954.

Así, el desarrollo de los programas y la bibliografía de lectura para los alumnos se encuentran, durante este período, confrontadas por el positivismo y el antipositivismo y, en algunos casos se observan programas mixtos donde se hace muy difícil señalar fronteras en cuanto a enfoques teóricos. Sin embargo, hemos verificado que existía un enfoque jurídico-normativo del desarrollo curricular en términos de legislación, y descripción, organización y gobierno del sistema escolar.

No obstante al analizar los programas del Prof. Rivarola se pueden leer los siguientes contenidos: "La laicidad escolar y la educación religiosa. La libertad de enseñanza y la obligación social de aprender. La educación de la mujer. La educación vocacional" (RIVAROLA, 1919), ampliando los ejes de análisis de la

\footnotetext{
${ }^{2}$ Espacio curricular que debían cursar todos los estudiantes del profesorado.
} 
cátedra. Además Rivarola incorpora en sus programas el estudio de la Educación Comparada al considerar como contenidos de estudios los sistemas educacionales de Estados Unidos, Alemania y Francia, entre otros.

Como temas que se repetirán de manera constante en los programas que hemos denominado precursores, podemos señalar el énfasis que toma la cuestión de la libertad de enseñanza en relación con la cuestión de la laicidad y la educación religiosa señalando la confrontación que se establecía sobre quién es el que ejerce la potestad para educar a las generaciones futuras.

Otro tema recurrente, es el acento en la educación nacionalista fundada en los escritos de Ricardo Rojas, fundamentalmente "La restauración nacionalista" de 1909 y la toma del modelo alemán del estudio de la historia, con el fin de tomar el fundamento para la conformación la unidad nacional tras la gran inmigración que traía consigo la multiplicidad de voces, culturas e ideologías.

\section{Surgimiento del campo: los espacios curriculares de política educativa}

La década de 1950 fue muy particular para las universidades nacionales de Buenos Aires y La Plata, ya que en Argentina se produce a "partir del año 1955 un parteaguas en la vida de la universidad argentina" (CHIROLEU, 2004, p. 1), iniciándose, por algo más de una década un proceso de modernización institucional. Considerando que la vida universitaria había sido profundamente afectada por los vicios cristalizados en varias décadas de desencuentros, intervenciones del poder político en su conducción y ausencia de proyectos de desarrollo integral. Así, a partir de la llamada Revolución Libertadora, se constata una transformación curricular y académica en ambas universidades.

En 1957 se aprueba en la Universidad de Buenos Aires el plan de Ciencias de la Educación que reemplaza al Profesorado de Pedagogía, en tanto en la Universidad Nacional de La Plata se considera al año 1959 como el de la nueva fundación de la carrera volviéndose a instaurar la denominación de Ciencias de la Educación. Tal como señala Tello (2013), en este período se produce:

El surgimiento de las "nuevas" carreras de ciencias de la educación en Latinoamérica con cierta sistematización para la enseñanza y la investigación, en sus diversas versiones: Política Educacional, Política y Legislación Escolar, Política y Educación Comparada, Política y Organización escolar y Política y Administración de la educación. Considerando el intercambio de nombres para las diversas cátedras entre 1950 y 1970 . Otro de los cambios que impacta sobre las "nuevas" carreras de ciencias de la educación y que fortalecieron la presencia y continuidad de las cátedras en las currículas universitarias se asocian al surgimiento de la corriente del desarrollismo latinoamericano en 
tanto los perfiles y funciones de los graduados en ciencias de la educación. Considerando que en esta corriente se requería, desde una perspectiva técnica, ejecutores de políticas educativas, analistas de la legislación escolar, planificadores educativos, técnicos, y analistas estadísticos en educación, entra otras funciones y, desde una perspectiva teórica: la vinculación con los “países desarrollados", la formación de recursos humanos y la formación profesional que permitiera identificar - en términos de ese período - las dificultades del subdesarrollo latinoamericano. (TELLO, 2013, p. 37).

Como afirma Carlino (1993), la incorporación de políticas desarrollistas desde los organismos internacionales buscaba la modernización de la sociedad y de sus instituciones, a partir de la legitimación científica desde las ciencias sociales. Estas políticas impactaron en las carreras de las ciencias de la educación en las universidades latinoamericanas:

En su carácter de organizaciones formadoras de elites dirigentes, las universidades debían ser transformadas para su contribución al desarrollo. Se plantea entonces un escenario más que interesante para indagar cuales eran los fundamentos teóricos de la materia Política Educativa en aquellos años en tanto que fueron los fundantes del campo. (CARLINO, 1993, p. 3).

Fue en los años 1953 y 1954 que se conforman las cátedras de "Política Educacional y Legislación Escolar", tanto en la UNLP a cargo del profesor Francisco Míguez como en la UBA a cargo de Albino Herrera.

En el año 1956, luego de lo que en la historiografía argentina se denominó Revolución Libertadora (1955), Américo Ghioldi es nombrado titular de las cátedras de "Política Educacional y Legislación Escolar" en ambas universidades, en las que permanecería hasta mediados de la década de 1960. Cabe señalar que en la Universidad de Buenos Aires en 1959, se modifica el espacio curricular de "Política educacional y Legislación Escolar" como parte de la transformación del plan de estudios de la carrera de ciencias de la educación que se llevó a cabo en 1957 transformándose en "Política Educacional y Educación Comparada" quedando, como mencionamos, bajo la responsabilidad del profesor Ghioldi.

En la búsqueda de los enfoques teóricos de las primeras cátedras, se realizó el análisis de los programas poniendo especial énfasis en la bibliografía de los mismos (véase Tabla 1 y 2 en Anexo). No es posible saber, debido al tiempo transcurrido, la selección real de los textos, ni la acentuación explicitada en las clases de los titulares de cátedra; sólo a través de la lectura de una gran parte del corpus bibliográfico y el análisis propio y de especialistas es posible develar los posicionamientos epistemológicos (TELLO, 2012) que asumían sus autores.

Se desprende, en primera instancia y tal como lo señala Tello (2013), en estos programas, un marcado posicionamiento jurídico - institucionalista: 
Dotada de las características principales de las ciencias políticas, surgía en la década de 1950 la política educativa como campo teórico, con una visión fuertemente centrada en la legislación y en algunos casos en educación comparada. Esta última no poseía el desarrollo epistemológico actual sino que, básicamente consistía en establecer ejes de comparación legislativos de diversos países y de la estructura del sistema educativo, entre otros temas de comparación lineal y casi descontextualizada. Pero sin duda respondía a la matriz analítica de las ciencias políticas en Latinoamérica con el enfoque jurídico-institucionalista. (TELLO, 2013, p. 37).

\section{En la Universidad Nacional de La Plata}

El primer programa de Política Educacional y Organización Escolar correspondiente a 1953 de la Universidad Nacional de La Plata a cargo del profesor Míguez, en el programa se observan cuatro bolillas de estudio. El primer contenido que se plantea se vincula a la demarcación del campo "La política educacional. Concepto, definición.” Es posible corroborar en cada una de las tres primeras unidades, dos núcleos de contenidos que fundamentan y adhieren a las políticas educativas de su tiempo. Por un lado la cuestión estatal: "La intervención del Estado en la orientación educacional", " Fundamentos jurídicos y sociales de dicha intervención"; en la bolilla 2, "Atribuciones de la nación, de las provincias y las facultades concurrentes en materia educacional", la comparación de las Constituciones de 1853 y del 1949; en la bolilla 3, la libertad de enseñanza en el estudio comparado (Inglaterra, España, Francia, Estados Unidos de Norte América) y por el otro sitúa como un actor clave a la Iglesia Católica como elemento fundante y de permanencia en el accionar educativo de la nación. Así, se señalan como contenidos: "Fray Antonio de San Alberto, Obispo de Tucumán", "Influencia benéfica de las órdenes religiosas. Franciscanos, dominicos, jesuitas y mercedarios", "La enseñanza de la religión. Planteo general y visión panorámica en nuestro medio". La cuarta bolilla trata la enseñanza de la religión, sus antecedentes, la discusión parlamentaria de la ley 1420 y el Decreto del 31 de diciembre de 1943, No 18.411, ratificado por el Congreso, mediante el cual se incluye la enseñanza religiosa en las escuelas públicas.

Es visible en este programa el marcado desplazamiento de tendencia teórica del humanismo laico y el cientificismo liberal por el humanismo y espiritualismo católico. Como señala Southwell (2003) una característica de la década de 1940 evidencia los postulados que sostenían los profesores incorporados en ese tiempo a la UNLP, como los presbíteros Octavio Derisi (quien fuera el primer Director del Departamento de Filosofía del cual dependía la carrera de pedagogía), Guillermo Blanco y Juan Sepich, que regían la Sección. 
El programa de 1954, también de Míguez, se inicia de igual manera que el de 1953 con la demarcación del campo. En la Bolilla 2 sugiere los posibles caminos que puede tomar la política educativa en tanto la teorización, la juridicidad y la facticidad. Luego aborda el tema de la libertad de enseñanza apoyándose en la Ley Nacional 934 de 1878 sobre educación privada y en la legislación comparada. En este programa no se hace alusión directa a la Iglesia Católica y a su presencia en la educación formal. Cabe destacar que las relaciones del gobierno peronista con la Iglesia Católica se habían deteriorado y como signo de esto podemos mencionar que Monseñor Octavio Derisi debió abandonar la Dirección de la Sección de Filosofía y que a nivel nacional, se derogó el decreto $n^{\circ} 18.411$ dejándose de dar educación religiosa en las escuelas públicas, medida impuesta en el año 1943.

Se incorpora en este programa la formación docente como así también la cuestión del nacionalismo, profundizándose en el enunciado de la bolilla siguiente al plantear como temática de estudio "el patriotismo". El nacionalismo y el patriotismo eran temas centrales en la ideología que sustentaba la cohesión de la nación desde del siglo anterior y se convertían en el objetivo central de la enseñanza escolar, particularmente la enseñanza de la historia y la geografía con el objetivo de crear la conciencia nacional. (HUARTE; VAN DER HORST, 2002).

La historia de la política educacional es expuesta a través de la bolilla VI y se centra en la influencia hispana en América, eje central de la educación patriótica planteada por Ricardo Rojas como modo de reconocer una identidad nacional hasta los tiempos posteriores a la Revolución de Mayo. Es particularmente significativo el hecho que no se mencione a la Ley 1420 del año 1884 a diferencia del programa de 1953.

Una bolilla completa toma la temática de la política educacional justicialista, acorde con la llamada Doctrina Nacional y su trascendencia nacional e internacional con especial interés en el análisis de la doctrina. Finaliza el programa con el enunciado sobre la descripción del sistema escolar y su relación con la política educacional vigente.

En el análisis de la bibliografía del programa es posible observar, que la mayoría de los textos que la conforman, tienen una antigüedad de cuarenta años o más de publicación. Algunos de las publicaciones que se conforman en nuestra investigación como componentes que nos ayudan a comprender la tendencia teórica del campo, se observa que se van entrecruzando en los programas los textos de Adolfo Posadas, "Política Pedagógica" de 1904. Este español, Doctor en Derecho que cursó estudios en el Instituto Libre de Enseñanza de Madrid donde, teniendo como mentor a Giner de los Ríos, se encolumnó dentro del 
krausismo español. Cabe señalar que este movimiento fue inspirado en el filósofo alemán Karl Christian Friedrich Krause, discípulo de Hegel, que tuvo una enorme influencia en España durante toda la segunda mitad del siglo diecinueve y que en nuestro país se lo puede incluir dentro del movimiento espiritualista.

Otro de los textos que Míguez incorpora en su programa es "Fundamentos científicos de la teoría de la constitución y de la política escolar", de Eduardo Spranger traducido por Lorenzo Luzuriaga. Este autor alemán, discípulo de Dilthey, sintetizó la filosofía clásica, el idealismo alemán y la corriente hermenéutica propiciada por su mentor. Inicia su obra con la siguiente afirmación "La creencia de que la política se obtenga en alguna parte de principios científicos o sistemas teoréticos es un error de gente culta" (SPRANGER, 1931, p. 5), para fundamentar a continuación la falsedad de los que afirman en los "círculos del magisterio": "que la ciencia de la educación puede ofrecer con sus medios fundamentaciones científicas unívocas" (SPRANGER, 1931, p. 5) para las medidas de la organización legislativa y las políticas escolares.

El autor afirma que el fin de este trabajo es:

[...] hacer intuitiva la relación entre teoría y práctica en el campo de las conexiones científico espirituales, pero del mismo modo debe explicar obligado por aquellas esperanzas puestas en una política escolar aplicable prácticamente de un modo aparentemente científico- el carácter problemático, desde el punto de vista de la ciencia, de una serie de tentativas que hasta ahora no han adquirido forma definitiva. (SPRANGER, 1931, p. 5).

El debate que plantea es el problema mismo de la configuración del campo, en tanto la imprecisión del nombre: Teoría de la Constitución Escolar, Teoría de la Administración Escolar, Teoría de la Organización Escolar o el más amplio: Política Escolar, como en los fundamentos y métodos donde "reina una falta completa de claridad" (SPRANGER, 1931, p. 7).

El texto prosigue explicitando las fuentes de la política escolar, así como también la conceptualización de términos, en tanto que define a la política escolar como:

[...] aquella parte de la política práctica que está dirigida a la situación de la escuela, o más en general: < de la instrucción pública > en el Estado, en lo cual el uso frecuente del lenguaje no establece a su vez ninguna diferencia entre si esta política es realizada por hombres de Estado responsables o por grupos en el Estado o por grupos aislados. (SPRANGER, 1931, p. 7).

Continúa haciendo un planteo y análisis del monopolio del Estado en la educación como así también sobre la libertad de enseñanza recuperando el recorrido histórico del concepto y sus diversas interpretaciones. 
Spranger va poniendo en evidencia su posición enfrentada al positivismo cuando afirma: "no existen normas de política escolar concretas derivables científicamente, no hay tampoco una ética concreta edificable sobre normas puramente científicas, evidentes" (SPRANGER, 1931, p. 60), así como también el posicionamiento puramente espiritualista, cargado de la conceptualización hegeliana, al que reiteradamente menciona "Queda, pues como médula de la educación el que actúe un espíritu sobre otro" (SPRANGER, 1931, p. 79).

Finaliza el libro con este concepto:

El problema capital del Estado moderno consiste en una protección de poder y de derecho sobre las oposiciones espirituales y sociales que cada día son más agudas. Aquel solo puede ser lo que Hegel sabía y quería: la unidad superior de lo diferente, en la que se concentra la multiplicidad de fuerzas valoradoras para asegurar la vida y la posesión espiritual cultural. (SPRANGER, 1931, p. 112).

Míguez asume desde sus programas la necesidad de una educación basada en el nacionalismo y el patriotismo fundamentándola a partir del texto de Rojas "La Restauración Nacional", en donde, como afirma Pulfer, en la presentación de la edición del 2010, el espiritualismo se afirma rechazando las doctrinas positivistas en búsqueda del "alma nacional". Rojas toma como uno de los puntos de partida el sustento hispano de la llamada generación del '98 entre los que se encontraba Unamuno, entre otros. Dentro de esta línea Míguez suma a la bibliografía un libro de su autoría "La patria y el patriotismo" exaltando las figuras de Monteagudo, San Martín, Sarmiento y Mitre casi como dioses de la patria y fundamenta su postura con la cita de Teófilo Sanjuán quien afirmaba que "Si la educación no es patriótica no es educación, así como el aire que respiramos sostiene nuestra vida biológica así la educación patriótica sostiene nuestra vida espiritual” (TEÓFILO SANJUÁN, 1923 apud MÍGUEZ, 1942, p. 141). Míguez (1942, p. 225) concluye: "Somos lo que somos, gracias a que nuestra patria es lo que es".

Como texto de fundamento filosófico, en la bibliografía se cita "Ensayo de una Filosofía Pedagógica" de Hovre, en él se sientan las bases del fundamento filosófico de la educación desde la doctrina católica y despliega una diatriba contra el naturalismo y el socialismo, a quienes considera con profundos análisis doctrinales como los verdaderos enemigos de la fe.

Otro de los autores de la bibliografía de este programa es Lorenzo Luzuriaga. Desde su activa producción desde la editorial Losada de Buenos Aires, ciudad a la que había arribado en medio de su exilio de la España franquista, Luzuriaga difunde los principios de escuela activa, pública, laica y unificada. Reconocido su posicionamiento dentro del socialismo, aborda los grandes 
nuevos temas de la pedagogía contemporánea. Entre sus referentes y escuelas de pensamiento, siguiendo a Gómez Martínez (2005), se encuentran la filosofía idealista alemana, particularmente a través de autores como Krause, Sanz del Río y Giner.

Luzuriaga entiende el carácter integral y armónico de la vida y de la educación espiritual, el sentido liberal-humanista de la educación inglesa, que observó en sus visitas a las instituciones británicas, donde se realzaban el aspecto humano, tolerante y vital en la actividad educativa y por último el sentido ético de la tradición filosófica española, representada sobre todo por el estoicismo de Seneca y que se manifiesta en el rigor y la austeridad en la conducta. Su lugar en la editorial Losada de Buenos Aires como el encargado de organizar y dirigir las publicaciones pedagógicas, permitió la difusión ininterrumpida de sus ideas.

Otro de los textos incluido en el programa del profesor Míguez es "La Política" de Aristóteles que nos indica uno de los presupuestos teóricos, la educación para el Estagirita es una función del Estado y más aún: es necesaria para el Estado. El fin de la educación es el reconocer el bien moral, no en el plano del mero conocimiento sino en su práctica a través de actos mediante la formación de hábitos que lleven a la vida virtuosa.

Las dos últimas unidades plantean el estudio la política educacional justicialista, acorde con la doctrina Nacional, su trascendencia nacional e internacional y la organización escolar actual.

Del programa y de su correspondiente bibliografía, de Míguez (1954) se puede inferir el entrecruzamiento del krausismo español a través del texto de Posadas, el espiritualismo de Spranger, quien fuera traducido por Lorenzo Luzuriaga, el escolanovismo y el nacionalismo argentino partiendo desde Ricardo Rojas, una tenaz postura católica que convive con autores reconocidamente socialistas, enmarcados en una opción aristotélica.

\section{En la Universidad de Buenos Aires}

En la Universidad de Buenos Aires, fechado en 1955, encontramos el primer programa de "Política Educacional y Organización Escolar" a cargo del Dr. Juan Albino Herrera. Los grandes temas, así como la bibliografía que toma, coinciden en general con los trabajados en La Plata por Míguez, pero a diferencia de este y dado que el programa fue confeccionado después de la llamada Revolución Libertadora, y habiéndose iniciado la "desperonización” de las universidades no se hace ninguna mención al justicialismo ni a Perón. No obstante, los enfoques teóricos y los temas en general son coincidentes. 
El programa de la materia consta de ocho bolillas. En la primera se establecen las definiciones correspondientes y responderían a esta bolilla los textos: "La Política", de Aristóteles, "Los fundamentos científicos de la teoría de la constitución y de la política escolares", de Eduardo Spranger; "Educación comparada", de Nicholas Hans; "Sociología de la Educación", de Fernando de Azevedo y "Pedagogía social y política", de Luzuriaga.

Debemos señalar que "Educación comparada "de Nicholas Hans, es considerado uno de los textos fundantes del campo de estudio homónimo, en sus páginas puede leerse la descripción de los factores que influyen en los sistemas educativos: naturales, religiosos, seculares. Acude al carácter nacional y señala cinco categorías fundamentales que constituyen una nación: unidad de raza, unidad de religión, unidad de lengua, territorio compacto, soberanía política.

"Sociología de la educación: introducción al estudio de los fenómenos pedagógicos y de sus relaciones con los demás fenómenos sociales" de Fernando de Azevedo es considerado el primer texto del campo mencionado en su título fundamentándose en el método sociológico de Durkheim.

En las unidades 3 y 4 encontramos, por ejemplo, a los siguientes autores y sus obras: "Fundamentos de la pedagogía cristiana" de Eustaquio Guerrero que es el comentario de la Encíclica "Divini Illius Magistri” del papa Pio XI del año 1929 en donde se establece el derecho de la Iglesia de educar y el derecho de la familia que concordaría con el de la Iglesia anterior al del Estado, se opone a cualquier tipo de educación naturalista que se contraponga a la formación sobrenatural cristiana, así como también a la educación sexual y a la coeducación que considera errónea y perniciosa. Rechaza la educación neutra o laica y proclama a la Iglesia como madre educadora.

El siguiente texto mencionado es "Legislación escolar y Ciencia de la Educación", un manuela con toda la legislación vigente del período, que se empleaba para los contenidos legislativos señalados en programa: "análisis de la Ley 934", "la Constitución de 1853 y la organización nacional", "las cuestiones jurisdiccionales", "la Ley de Educación Común 1420" y "la descripción de la enseñanza media y universitaria".

Luego, se menciona, el texto de Amanda Labarca, "Bases para una política educacional" de 1944. En este texto la autora presenta una lectura de la realidad educativa de América que denota una mirada crítica sobre las razones de la situación de dependencia y sometimiento del continente. Esta educadora chilena proporciona una mirada, novedosa para el período, aunque comenzaban a emerger este tipo de reflexiones latinoamericanas, allí explica algunas cuestiones propias de la realidad latinoamericana como la falta de identidad, el atropello y el sometimiento. En las páginas siguientes hace una severa crítica a 
la Iglesia Católica a la que acusa de "identificar su suerte con la de los grupos aristocráticos" (LABARCA, 1944, p. 14). Consciente de la necesidad de un cambio al modificarse el sentido de la escuela a la que hoy se le requiere mucho más que la formación intelectual, ve como salida el movimiento de la escuela nueva. En el capítulo 2 juzga que el mestizaje inconcluso, no solo de sangre, sino de costumbres, intereses e ideales, que existe en los países sudamericanos, no ha permitido la homogeneidad social impidiendo la formación de la conciencia nacional, señala que en la clase media ya se está dado pero que esta no tiene el suficiente poder en la cuestión económica y pública. Continúa con una denuncia feroz sobre la ética sexual de la población de los países sudamericanos. Otra de las críticas que pronuncia es acerca de las constantes rupturas con el pasado y la búsqueda de diferenciarnos de nuestros países vecinos, describe el complejo de inferioridad que hace que se pretenda hacer una nueva Europa en vez de reconocerse como indoamericanos y esto hace que se valore exageradamente lo extranjero.

El capítulo 3 se titula "Las coordenadas de una política educacional", afirma la autora que:

la política educacional ha de afianzarse en estos tres sillares: sociedad, ideales de vida (que son idénticos a los de la educación) y condiciones sicofisiológicas de esta niñez, adolescencia y juventud. ¿Qué precisa nuestra sociedad de su generación joven? ¡Qué necesita vitalmente’ ¿Y qué puede esperarse de la reacción juvenil? (LABARCA, 1944, p. 60).

La educación nacional-espiritualista se ve cubierta con el libro de Juan Mantovani “Épocas y hombres de la educación argentina “. Este es un texto que como se señala en el prólogo reúne "diversos escritos sobre figuras centrales y aspectos de nuestra historia de la educación", tales como Rivadavia, Mitre y Sarmiento quienes, afirma, dieron su vida por la nacionalidad argentina. Encendidos panegíricos sobre estas figuras a las que suma a Amadeo Jacques, describen héroes sin fallas ni errores. Suma un capítulo sobre la segunda enseñanza y la universidad en relación a la formación nacional que es un relato sin contradicción y de modo lineal sobre el desarrollo de los niveles educativos en nuestro país.

No debemos dejar de lado la trascendencia de la figura, la trayectoria y el pensamiento del Mantovani. Una de las frases con las que se puede ilustrar su pensamiento es que la educación "toma al hombre en su unidad formada de espíritu y vida y en la complejidad histórico cultural de su época y de su medio" (MANTOVANI, 1950 p. 21). Stramiello (2011) explica que Mantovani propone a la educación como un proceso exclusivamente humano, que tiene como fin esencial al hombre pleno sin caer en un reduccionismo antropológico, necesita de las distintas disciplinas que tomen los distintos aspectos del hombre, 
el biológico el psíquico, el espiritual, el ético. Una de las materias necesarias es la filosofía y explícitamente la antropología filosófica, como punto de partida de las propuestas pedagógicas. Desde una visión antipositivista bajo la influencia de Alejandro Korn y Coriolano Alberini entre otros, sumada a la lectura de Kant, Gentile y Radice y a los encuentros con Ortega y Gasset, Miguel de Unamuno y Albert Einstein lo convirtieron en uno de los representantes del antipositivismo en el ámbito educativo. También se deben tener en cuenta las influencias de Scheler y Spranger asociada al ideal del hombre todo y los valores objetivos. La actividad desbordante de Mantovani lo convierte en uno de los personajes con mayor influencia tanto desde su actividad en las universidades o en la época de proscripción, desde la Escuela de Libre Enseñanza así como también en su trascendencia a nivel internacional a través de conferencias que dicto por toda América y en sus trabajos para la UNESCO.

\section{El Profesor Ghioldi}

Un apartado especial merece el desarrollo y la actividad del profesor Ghioldi dado que se desempeñó como profesor de políticas educativas en ambas universidad a poco de crearse las cátedras.

Tanto en la Universidad Nacional de Buenos Aires como en la Universidad Nacional de la Plata asume la cátedra de Política Educacional y Legislación Escolar en 1956, en las que permanecerá hasta 1965, periodo en el que se consolida el campo de la Política Educativa en las dos universidades.

Viana (2011) caracteriza la postura de Ghioldi afirmando que abreva de tres fuentes, del laborismo ingles la fundamentación ética de un modelo de bienestar democrático, del socialismo francés toma la orientación de Jean Jaures que no postula al socialismo como una garantía cientificista del advenimiento de la sociedad de libre cooperación, sino que reposiciona al socialismo, como un ideal regulativo, a partir de la renovación de Alejandro Korn que tiene un posicionamiento más filosófico que doctrinario; la tercera influencia teórica refiere a Benedetto Croce del que toma el concepto de historia como aventura de la libertad.

De extracción socialista, se impuso como líder parlamentario en la década del '30, convirtiéndose a finales de la década del '40 en el principal referente intelectual de su partido y en una de las más potentes voces del antiperonismo, con una intensa crítica al peronismo como legislador y desde la "La Vanguardia", órgano oficial de su partido de quien era director.

Para Ghioldi el peronismo era signo de totalitarismo que pretendía que el hombre se entregara al estado a modo de lo hicieron el nazismo y el fascismo. Lo cual consideraba como inaceptable dado que estaban inspirados 
en un existencialismo confuso, de origen germánico que renegaba de la razón y olvidaba los derechos del hombre.

En 1947, “La Vanguardia”, periódico en que denostaba en cada edición al gobierno de Perón, es clausurada y Ghioldi es detenido y finalmente debe emigrar a Uruguay. En 1951 participó del frustrado golpe del general Menéndez como así también fue un activo propiciador de la llamada Revolución Libertadora, junto a Alfredo Palacios, Alicia Moreau de Justo, Nicolás Repetto, Ramón Muñiz, entre otros, que finalmente derrocó a Perón en 1955.

Ghioldi representa al socialismo argentino que se presenta como los defensores de la normatividad estatal, fundándose en la constitución y al orden impuesto por ella como el único camino válido para el orden social. Puiggrós (1983, p. 97) lo señala como "el último de los grandes positivistas en el registro político-pedagógico" tornándose como consecuencia hacia la derecha del espectro político, levantando las banderas del laicismo y la Constitución. Cabe destacar que desde los años 40, Ghioldi se acercó a una postura pronorteamericana "los Estados Unidos representaban una síntesis contemporánea entre libertad y justicia social" (PUIGGRÓS, 1983, p. 98). Propuso una lectura del liberalismo en términos de libertades civiles y valores civilizatorios. Adhería a una pedagogía de la libertad que requería una religión laica siendo la Constitución el móvil de la historia que condensaba la vida y el pensamiento de la nación.

Esta cuestión se dio por la influencia del Teachers College de la Universidad de Columbia, donde Amanda Labarca, autora de "Bases para una política educacional", como Ernesto Nelson, con su presencia permanente en la bibliografía de la totalidad de los programas de Ghioldi, habían asistido a clases con Dewey y donde habían nacido la nuevas formas de comprender la Educación Comparada de la mano de Kandel y Hans.

Ghioldi, optó desde sus cátedras seguir la línea de pedagogía constitucional, apoyándose en la Constitución de 1853, dirigida hacia el laicismo, la constante relectura de la Ley 1420 a la que no le formulaba objeción alguna y los relatos históricos sin ningún margen de crítica a quienes considera modelos: Sarmiento, Rivadavia, Mitre. También se puede observar en los programas de sus cátedras la presencia constante de Luzuriaga como referente del progresismo pedagógico. Sin dudas que su reconocimiento a Krause y Spranger como sustento filosófico fueron influencias clave en el desarrollo y confección de los programas de política educativa, del mismo que se puede observar como en 1959 realiza la incorporación en su programa de textos de Rodolfo Mondolfo quien aporta la justificación filosófica del trabajo para la superación del hombre.

Ghioldi incluye textos propios como parte de la Bibliografía del año 1959 en los que sintetiza, de cierta manera su pensamiento. Por ejemplo "Formación 
de la escuela argentina", colección de textos breves que resumen una serie de conferencias dadas por Ghioldi a partir del cincuentenario de la ley 1420. Otro de los textos propios que incluye en el programa es un proyecto de resolución presentado frente al Congreso de la Nación durante su mandato como diputado por el partido Socialista que dio en llamar "Por la Educación común, nacional e individual. Federalización de la instrucción Primaria". El fin de este proyecto era la eliminación del analfabetismo y la deserción escolar. También incluye un proyecto de ley que denomina "Oportunidad de formación para los jóvenes. Normas de aprendizaje técnico". El texto de este proyecto de ley proponía la creación de un departamento nacional de trabajo con una división de aprendizaje, siendo su función la formación integral de aprendices. Estaba dirigido a jóvenes, varones o mujeres, de 16 a 17 años para que, mediante la firma de un contrato de aprendizaje, se les diese a los jóvenes la posibilidad de aprender un oficio en las diferentes fábricas. El fundamento era la nacionalización de la mano de obra calificada entre los jóvenes nativos.

A través de los años, en los sucesivos programas, Ghioldi, incorpora las publicaciones de organismos internacionales como la Organización Internacional de Educación, la UNESCO y la OIT, añadiendo temas de análisis como por ejemplo la relación de la educación con los nuevos modos de trabajo.

\section{A modo de cierre}

En la formación del campo de la Política Educativa, a partir de los procesos pedagógicos que se cristalizan en los programas de estudio como parte del cuadro de las ciencias de la educación en la década de 1950, se observan tendencias teóricas que sin dudas, conviven entre sí:

a. la tradición nacional, muy marcada en los programas de Míguez a través de la exaltación de los valores de la patria y el patriotismo fundada en el proceso histórico y civilizatorio;

b. la fuerte presencia del progresismo pedagógico a través de la profusa presencia de los textos de Lorenzo Luzuriaga e

c. la influencia del krausismo español que ya estaba inserto en la cultura pedagógica argentina desde los tiempos de Sarmiento y el sustento del sentido de la política educacional de Spranger.

Hemos podido comprobar que cada uno de los programas estuvo atravesado por la contingencia histórica, el despliegue de la doctrina justicialista entre 1953 y 1954 en el programa de Míguez, la ausencia referencial a la coyuntura del momento en el programa de Herrera, la visión contraria a los gobiernos totalitarios y la opción por un modelo norteamericano en Ghioldi. 
Por otra parte se advierte en los programas analizados la presencia del contenido que refiere a la Iglesia Católica como actor constante en la tensión dada en la lucha por la influencia en el proyecto pedagógico de la nación, hasta la inclusión de "las iglesias" en plural, en el programa de 1959 de la Universidad Nacional de La Plata en función de la aceptación del ingreso al campo de discusión de otros cultos que van haciéndose en la vida de la sociedad de ese tiempo.

Asimismo, en cada uno de los programas, se constata la presencia del enfoque centrado en la legislación y en la educación comparada, sin embargo no se desprende del análisis de las bibliografías una mirada crítica sino simplemente el reconocimiento de la legislación que regulaba el sistema educativo nacional y de los sistemas educativos norteamericanos y europeos, particularmente de Francia, Inglaterra y Alemania.

Podemos mencionar algunos temas cuya ausencia posee cierta significatividad desde nuestra perspectiva, pero que, sin dudas, no era parte del debate y la reflexión en el surgimiento del campo, nos referimos a la realidad latinoamericana -que tan solo es tenida en cuenta con el texto de Amanda Labarca - la adhesión absoluta al presupuesto de que la educación es monopolio del Estado, sin incluir en los procesos educativos otros actores clave, y la ausencia de descripciones y análisis de las realidades contemporáneas. O dicho de otro modo, estas realidades solo se pueden visualizar a través de los documentos seleccionados - principalmente en los programas de Ghioldi - provenientes de organismos internacionales.

Imbricados en la genealogía de nuestro campo de estudio, las diferentes tensiones de los posicionamientos epistemológicos e ideológicos que se entrecruzan en los programas analizados nos dan la pauta de temas aún no resueltos. Con el pasar de los años nuevas corrientes ideológicas se irán sumando en la historia de la construcción del campo de la política educativa, sin desplazar del todo las cuestiones seminales que se hacen presentes, sin estar debidamente explicitadas, en las contradictorias políticas educativas que se muestran como novedosas a lo largo de los últimos 60 años de historia educativa.

\section{Referencias}

BOURDIEU, P. Campo de poder, campo intelectual. Tucumán, Argentina: Montressor, 2002.

CARLINO, F. Transformaciones de la carrera de ciencias de la educación de la UBA. Un recorrido histórico de algunos problemas actuales. Revista del Instituto de Investigación en Ciencias de la Educación, año II, n. 3, p. 49-58, 1993. 
CHIROLEU, A. Luces, sombras y matices de la primera modernización universitaria. In: ENCUENTRO NACIONAL Y I LATINOAMERICANO LA UNIVERSIDAD COMO OBJETO DE INVESTIGACIÓN, 4., 2004, Tucumán. Anais... Tucumán, 2004.

GÓMEZ CAMPO, V; TENTI FANFANI, E. Universidad y profesiones. Buenos Aires: Miño y Dávila, 1989.

GÓMEZ MARTÍNEZ, J. L. Lorenzo Luzuriaga. En: Proyecto ensayo hispánico. 2005. Disponível em: <http://www.ensayistas.org/antologia>. Acesso em: 1 nov. 2013.

HUARTE, G; VAN DER HORST, C. Historiografía y la enseñanza de la historia en Argentina. In: CASTRO, M. (Comp.). Visiones latinoamericanas: educación, política y cultura. México: Plaza y Valdez, 2002. p. 227-251.

MANTOVANI, J. Épocas y hombres de la educación argentina. Buenos Aires: El Ateneo, 1950.

MÍGUEZ, F. La patria y el patriotismo. Quilmes: A.B.C., 1942.

MÍGUEZ, F. Política Educacional y Organización Escolar. Programa. La Plata: Universidad Nacional de La Plata, 1954. p. 1-6.

PALAMIDESSI, M.; GALARZA, D.; CARDINI, A. Un largo y sinuoso camino: las relaciones entre la política educativa y la producción de conocimientos sobre educación en Argentina. In: PALAMIDESSI, M., SUASNABAR, C.; GOROSTIAGA, J. (Comps.). Investigación educativa y política en América Latina. Buenos Aires: Noveduc, 2012. p. 113-140.

PUIGGRÓS, A. (Dir.). Dictaduras y utopías en la historia reciente de la educación argentina (1955 -1983). Buenos Aires: Galerna, 1983.

RIVAROLA, H. Ciencia de la Educación. Programa. La Plata: Universidad Nacional de La Plata, 1919. p. 1-5.

SIRVENT, M. T.; RIGAL, L. Algunas notas sobre teoría y empiria en investigación educativa. 2007. (Mimeo).

SOUTHWELL, M. Psicología experimental y Ciencias de la Educación. Notas de Historia y Fundacionales. Universidad Nacional de La Plata, 2003.

SPRANGER, E. Fundamentos científicos de la Teoría de la Constitución y de la Política Escolar: la pedagogía social y política. Madrid: Publicaciones de la Revista Pedagógica, 1931.

STRAMIELLO, C. Juan Mantovani. La educación como consolidación de la humanidad. En Educadores en America Latina y el Caribe del siglo XX al siglo XXI. Colombia: Soto Arango y otros Editores, Ediciones Doce Calles S. L., Tunja, 2011.

TELLO, C. Las epistemologías de la política educativa: vigilancia y posicionamiento epistemológico del investigador en política educativa. Práxis Educativa, Ponta Grossa, v. 7, n. 1, p. 53-68, jan./jun. 2012. DOI: 10.5212/PraxEduc.v.7i1.0003

TELLO, C. Las epistemologías de la política educativa: notas históricas y epistemológicas sobre el campo. In: TELLO, C. (Comp.). Epistemologías de la política educativa: posicionamientos, perspectivas y enfoques. Campinas: Mercado de Letras, 2013. p. 23-68.

VIANA, J. M. Pedagogía y Política en el Antiperonismo de Américo Ghioldi. Intersticios de la política y la cultura latinoamericana: los movimientos sociales, Córdoba, n. 1, p. 88-93, 2011. 


\section{Fuentes}

ARISTÓTELES. La política. México: Porrúa, 1991.

AZEVEDO, F. de. Sociología de la educación: introducción al estudio de los fenómenos educativos y de sus relaciones con los demás fenómenos sociales. México: Fondo de Cultura Económica, 1942.

DE HOVRE, Fr. Ensayo de Filosofía Pedagógica. Madrid: Ediciones Fax, 1945.

GHIOLDI, A. Política educacional en el cuadro de las Ciencias de la Educación. Buenos Aires: Losada, 1972.

GHIOLDI, A. Formación de la escuela argentina. Buenos Aires: Imprenta Federación Gráfica Bonaerense, 1934. (Sociedad Luz, Serie II, Tomo X, n. 191).

GHIOLDI, A. La educación común, nacional e individual: federalización de la instrucción primaria. Buenos Aires: Imprenta del Congreso, 1941.

GHIOLDI, A. Oportunidad de formación para los jóvenes, normas de aprendizajes técnicos. Buenos Aires: Imprenta del Congreso, 1942.

GHIOLDI, A. Política educacional en el cuadro de las Ciencias de la Educación. Buenos Aires: Losada, 1972.

GUERRERO EUSTAQUIO. Fundamentos de la pedagogía cristiana. Buenos Aires: Poblet, 1947.

LABARCA, A. Bases para una política educacional. Buenos Aires: Losada, 1944.

LUZURIAGA, L. La educación de nuestro tiempo. Buenos Aires: Losada, 1957.

LUZURIAGA, L. Pedagogía social y política. Buenos Aires: Losada, 1954.

MANTOVANI, J. Épocas y hombres de la educación argentina. Buenos Aires: El Ateneo, 1950.

MÍGUEZ, F. La patria y el patriotismo. Quilmes: A.B.C., 1942.

MíGUEZ, F. Política Educacional y Organización Escolar. Programa. La Plata: Universidad Nacional de La Plata, 1954. p. 1-6.

NELSON, E. Nuestros males universitarios. Buenos Aires: El Ateneo, 1919.

PÍO XI. Carta Encíclica Divini Illius Magistri de S.S. Pío XI, sobre la Educación Cristiana. 3 de diciembre de 1929. Disponível em: <http://www.cafaalfonso.com.ar/ descargas/diviniilliusmagistri.pdf>. Acesso em: 1 nov. 2013.

RIVAROLA, H. Ciencia de la Educación. Programa. La Plata: Universidad Nacional de La Plata, 1919. p. 1-5.

ROJAS, R. La restauración nacionalista de Ricardo Rojas: presentación Darío Pulfer. La Plata: UNIPE, Editorial Universitaria, 2010.

SPRANGER, E. Fundamentos científicos de la Teoría de la Constitución y de la Política Escolar: la pedagogía social y política. Madrid: Publicaciones de la Revista Pedagógica, 1931. 


\section{ANEXO}

Tabla 1 - Programas analizados de la Universidad Nacional de Buenos Aires

\begin{tabular}{c|c|c}
\hline Años & Materia & Docente \\
\hline 1917 & Ciencia y Educación & Carlos Octavio Bunge \\
\hline 1918 & Ciencia de la Educación & Carlos Octavio Bunge \\
\hline $1919-1920$ & Ciencia de la Educación & Horacio C. Rivarola \\
\hline 1922 & Ciencia de la Educación & Juan P. Ramos \\
\hline $\begin{array}{c}\text { Desde } 1926 \\
\text { a } 1946\end{array}$ & Metodología y Legislación Escolar & Julio del C. Moreno \\
\hline $\begin{array}{c}\text { Desde } 1946 \\
\text { a } 1954\end{array}$ & Seminario: Organización Escolar & Juan Cassani \\
\hline 1955 & Política Educacional y organización Escolar & Juan Albino Herrera \\
\hline 1956 & Política Educacional y Organización Escolar & Américo Ghioldi \\
\hline $\begin{array}{c}\text { Desde } 1957 \\
\text { a } 1959\end{array}$ & Política Educacional y Educación comparada & Américo Ghioldi \\
\hline
\end{tabular}

Tabla 2 - Programas analizados de la Universidad Nacional de La Plata.

\begin{tabular}{c|c|c}
\hline Años & Materia & Docente \\
\hline 1938 & $\begin{array}{c}\text { Legislación Escolar } \\
\text { (programa sintético) }\end{array}$ & Juan Cassani \\
\hline $\begin{array}{c}1953 \\
\text { (fragmento) }\end{array}$ & Política Educacional y Organización Escolar. & Francisco Míguez \\
\hline 1954 & Política Educacional y Organización Escolar. & Francisco Míguez \\
\hline $\begin{array}{c}\text { Desde } 1956 \\
\text { a } 1959\end{array}$ & Política Educacional y Organización Escolar. & Américo Ghioldi \\
\hline
\end{tabular}




\title{
Programa de Política Educacional y Organización Escolar Prof. Francisco Míguez \\ Año 1953. Universidad Nacional de La Plata
}

\author{
BOLILLA I
}

$1^{\circ}$.- La Política Educacional.- Concepto y definición.

Intervención del Estado en la orientación educacional. Fundamentos Jurídicos y Sociales de dicha intervención.

Función Social y función estatal.

Distintos elementos en el Derecho Comparado.

$2^{\circ}$.- Orientación en materia educativa seguida por España en América.

Distintos criterios interpretativos. Leyes de Indias.

La enseñanza en sus tres aspectos.

BOLILLA II

$1^{\circ}$.- Atribuciones de la nación en materia educacional.

Atribución de las provincias.

Facultades concurrentes.

Estudio comparativo de la Constitución del ‘ 53 y el ‘ 49.

Fray José Antonio de San Alberto, Obispo de Tucumán.

BOLILLA III.

$1^{\circ}$.- Libertad de enseñanza en el Derecho Comparado (España - Inglaterra Alemania - Francia - Estados Unidos de Norte América).

Sistemas seguidos en nuestro país, antecedentes históricos, fundamentos, aspecto constitucional. Estudio exhaustivo de la Ley 934.

$2^{\circ}$.- Influencia benéfica de las órdenes religiosas. Franciscanos, dominicos, jesuitas y mercedarios.

BOLILLA IV

$1^{\circ}$.- La enseñanza de la religión. Planteo general y sesión panorámica en nuestro medio. Antecedentes hasta 1884. Ley 1420, su discusión parlamentaria.

Decreto del 31 de diciembre de 1943, $\mathrm{N}^{\circ}$.- 18.411. Ley 12.978 y decreto reglamentario, $\mathrm{N}^{\circ} 13.182$. 
$2^{\circ}$.- Prédica cultural por los periódicos.- Belgrano. Cabello y Mesa Vieytes.

Sin bibliografía. (Programa de Cátedra 1953. UNLP. Prof. Míguez)

\section{Programa de Politica Educacional y Organización Escolar Prof. Francisco Míguez}

\section{Año 1954. Universidad Nacional de La Plata}

Bolilla I. La política educacional: concepto, extensión. Factores de la política educacional: clases, enunciación.

Bolilla II. Teoricidad, juridicidad, facticidad. Análisis de esos órdenes, definiciones, ejemplos.

Bolilla III. Los problemas de la libertad de enseñanza: planteo, soluciones; Legislación comparada. Ley Nacional n 934, antecedentes, debates, crítica.

Bolilla IV. El problema de la formación cuerpo docente: grados y especialidades. El cuerpo docente y la nacionalidad.

Bolilla V. El problema del nacionalismo y del patriotismo. La enseñanza de la historia con relación a este problema.

Bolilla VI. La política educacional seguida por España en América: su evolución. Valoración crítica. Legislación.

Bolilla VII. La política educacional en nuestro país después de la Revolución de Mayo: hombres y épocas características.

Bolilla VIII. La política educacional justicialista, acorde con la Doctrina nacional: definición de ésta, análisis. Trascendencia nacional e internacional del Justicialismo Argentino. Carácter de la Doctrina.

Bolilla IX La organización escolar actual en nuestro país: descripción. Relación existente entre la política educacional y la organización escolar actuales.

\section{Bibliografía Fundamental}

Adolfo Posadas "Política pedagógica"

Manuel H. Solari "Política educacional argentina"

Aristóteles "La Política"

Eduardo Spranger "Fundamentos científicos de la teoría y de la constitución y de la política educacional"

Bernardo Spaventa "La liberta dínsegnamento" 
Ley Nacional $N^{\circ} 934$

Ernesto O’Dena "Debates parlamentarios sobre instrucción pública”

Carmelo Zingoni "Formación universitaria del maestro"

Enrique Pollero "Segundo Congreso Nacional de Profesores de enseñanza secundaria" (La formación del profesor)

Lorenzo Luzuriga, "La preparación de los maestros"

R. Rivarola, "Formación del profesorado secundario, Tm I, pág. 129.

Joaquín V. González, "Política Universitaria"

Ricardo Rojas, "La restauración Nacional"

Carlos O Bunge, "La enseñanza nacional"

Ernesto Quesada, "La enseñanza de la historia en las universidades alemanas"

Francisco Míguez, "La patria y el patriotismo"

Teofilo Sanjuán, "La enseñanza de la historia en la escuela primaria"

Fra. D Hovre "Ensayo de filosofía pedagógica"-Poblet, Bs. As. 1947.

Enciclopedia de la Educación Moderna,-Losada, Año 1946, Tm.II pg. 145.

Ott Willmann, “Teoría de la formación humana” 'Tm I pág. 363; Tm.II pág. 38, 307, 333, 339, 363, 173, 446, 459.

Leyes de Indias: Ley IV, Título XIII, Ley V, Título XIII, Ley XI, Título XXIII, Ley XIV, Título XXIII, Ley XXXIX, Título XII.

“Monitor de la Educación Común”, jul. A set. 1949. - Abel Chanetón

"La instrucción primaria en la época colonial" - Solarzano

"Política Indiana"

El resto de la bibliografía será suministrada en clase. Cada alumno deberá entregar una monografía en el tiempo establecido por las ordenanzas vigentes. (Programa de cátedra de 1954. UNLP. Profesor titular Francisco Míguez)

\section{Programa de Política Educacional y Organización Escolar Profesor interino ad honorem: Dr. Juan Albino Herrera Año 1955. Universidad Nacional de Buenos Aires}

I.Concepto y extensión de la materia. Factores y agentes de la política educacional. Legislación y educación. 
II. El estado y la libertad de enseñanza. Legislación argentina.

III. La religión y la educación. Posicionamiento de la iglesia católica. Su proyección en la Argentina.

IV. La política educacional argentina. El período hispánico. La constitución de 1853 y la organización nacional.

V. Organización política del país. La nación y las provincias: sus atribuciones en materia educativa. El Ministerio de educación, organización y competencia.

VI. La instrucción pública argentina. La educación primaria. La ley de educación común: antecedentes, debates y soluciones.

VII. La enseñanza media argentina; sus fines, contenidos y organización. La universidad y la enseñanza preparatoria.

VIII. La enseñanza superior argentina; sus fines y realizaciones. Universidades, academias e institutos. Organización universitaria; leyes y estatutos. La autonomía universitaria.

\section{Bibliografía:}

Aristóteles. La política. Buenos Aires. Espasa Calpe;

Spranger, Eduardo, Los fundamentos científicos de la teoría de la constitución y de la política escolar, Madrid, Revista de Pedagogía, 1931;

Hans, Nicholas, Educación comparada, Buenos Aires, Nova, 1953;

De Havre, F. Ensayo de Filosofía Pedagógica. Buenos Aires, Poblet, 1947;

Acevedo, Fernando de. Sociología de la Educación, México, Fondo de Cultura, 1954;

Luzuriaga, Lorenzo, Pedagogía social y política, Buenos Ares, Losada, 1954;

Guerrero, Eustaquio. Fundamentos de la pedagogía cristiana, Buenos Aires, Poblet, 1947;

Rivarola, Horacio, Legislación escolar y ciencia de la educación, Buenos Aires, El Ateneo, 1944;

Labarca, Amanda, Bases para una política educacional, Buenos Aires, Losada, 1944;

Mantovani, Juan, Épocas y hombres de la educación argentina, Buenos Aires, El Ateneo, 1950;

Probst, Juan, La educación en la República Argentina durante la época colonial, Buenos Aires, 1924;

Ramos, Juan P., Historia de la instrucción primaria, Buenos Aires (Programa de cátedra de_1955. UBA. Profesor Juan Albino Herrera).

Recebido em 26/11/2013

Versão final recebida em 08/04/2014

Aceito em 12/04/2014 